\title{
tic\&société
}

Vol. 5, $n^{\circ} 1 \mid 2011$

TIC et genre

\section{TIC et genre : des regards multiples}

\section{Patricia Vendramin}

\section{(2) OpenEdition}

Journals

Édition électronique

URL : http://journals.openedition.org/ticetsociete/938

DOI : 10.4000/ticetsociete.938

Éditeur

Association ARTIC

Référence électronique

Patricia Vendramin, "TIC et genre : des regards multiples », tic\&société [En ligne], Vol. 5, n 1 | 2011,

mis en ligne le 05 octobre 2011, consulté le 19 avril 2019. URL : http://journals.openedition.org/

ticetsociete/938; DOI : 10.4000/ticetsociete.938 
tic\&société - 5 (1), 2011

\section{TIC et genre : des regards multiples}

\section{Patricia VENDRAMIN}

Directrice de recherche à la Fondation Travail-Université (FTU, Namur, Belgique)

Professeure à l'Université de Louvain (UCL)

FTU, Rue de l'Arsenal, 5, B-5000 Namur pvendramin@ftu-namur.org

Docteur en sociologie. Directrice de recherche à la Fondation Travail-Université à Namur (Belgique). Professeure invitée à l'Université de Louvain (UCL) où elle enseigne la sociologie et les sciences du travail. Ses recherches et publications concernent: les mutations du travail ainsi que l'exclusion et l'inclusion dans la société de l'information. 
TIC et genre : des regards multiples

A l'heure où les technologies de l'information et de la communication (TIC) prennent une place grandissante dans la vie quotidienne et professionnelle, des différences liées au genre viennent s'inscrire dans la conception, l'usage, l'appropriation et la maîtrise de ces outils stratégiques.

Loin de souffrir d'un quelconque handicap technologique, les femmes et les jeunes filles, dans leur vie quotidienne ou scolaire, sont des utilisatrices aussi averties que leurs homologues masculins. Les écarts qui existaient au début de la diffusion de ces outils s'amenuisent fortement avec la banalisation des TIC dans les activités journalières. La disparité numérique a toutefois d'autres facteurs explicatifs que le genre, comme le revenu ou le niveau de formation. Dans le monde professionnel, les femmes sont également des utilisatrices confirmées des TIC, dans la plupart des branches d'activités où elles sont actives. Néanmoins, elles restent particulièrement minoritaires dans les métiers des TIC proprement dits. En effet, sauf exception, partout en Europe et en Amérique du Nord, la proportion de postes occupés par des femmes dans ces métiers est faible, de même que la proportion de femmes dans les filières scolaires conduisant à ceux-ci (Vendramin et Valenduc, 2006 ; Vendramin, 2004, 2005). Elles sont donc largement absentes des lieux où se conçoivent et se maîtrisent les enjeux des TIC (conception, gouvernance...). Manque d'intérêt ou éviction insidieuse, divers facteurs se conjuguent pour expliquer ces différences.

Plusieurs hypothèses sont habituellement avancées pour tenter d'expliquer les dissimilitudes de genre dans le rapport aux TIC. Ces hypothèses sont spécifiques aux TIC et se distinguent, en partie, des hypothèses relatives à d'autres champs techniques. La perspective sociologique de la relation entre femmes et technologies s'intéresse aux processus de socialisation sans trop questionner la technologie en tant que telle. Elle développe des hypothèses autour de l'éducation et de la formation, des conditions de travail, de la culture de métier et de l'ensemble du monde professionnel des TIC, considéré comme défavorable pour les femmes.

Peterson (2010), par exemple, analyse les stratégies des femmes dans des environnements d'expertise technique, dont particulièrement les métiers des TIC. Pour les femmes, conclut cette auteure, afficher une faible confiance en soi dans les matières techniques est une stratégie pour se faire accepter dans des contextes professionnels marqués par un idéal de travail masculin. En adoptant cette attitude, les femmes ne menacent pas la suprématie mâle et se font 
accepter. Par contre, quand les femmes s'adaptent à cet idéal de travail masculin, montrent de l'ambition, de l'initiative et de l'esprit d'entreprendre, elles suscitent la désapprobation à la fois des hommes mais aussi des autres femmes. L'idéal professionnel masculin contribue ainsi à maintenir les femmes dans des positions subordonnées et limite leurs possibilités de carrière dans ces secteurs.

Les cultural studies, en particulier les gender studies, venues des pays anglo-saxons, ont amené d'autres courants de recherche et ouvert un questionnement sur la technologie elle-même. Un ensemble d'hypothèses associe la technologie à l'univers masculin (rationalité, compétition, domination...) et étudie la relation ambivalente des femmes aux TIC, le caractère hostile de la culture informatique pour les femmes, les aptitudes différenciées par rapport aux hiérarchies statiques et aux réseaux dynamiques. Turkle (1997) oppose l'approche féminine "non linéaire " de l'ordinateur, la " maîtrise douce » et le bricolage des femmes à la "maîtrise dure » des hommes, la pensée abstraite et linéaire. Des pratiques sexuées sont mises en évidence dans les approches différentes de la programmation, l'orientation des jeux vers les garçons, les styles de communication, le contenu et le design des sites, les scripts sexués. Royal (2009), par exemple, analyse les espaces dédiés aux femmes sur Internet et constate comment ceux-ci tendent à reproduire les stéréotypes de genre en vigueur dans les magazines féminins.

Des auteurs comme Wajcman (1991) montrent que l'identification entre les hommes et les machines n'est pourtant pas immuable mais résulte de processus idéologiques et culturels. Son parcours dans la littérature sociologique et féministe relative à la technologie la conduit à mettre en évidence le biais masculin existant dans la manière de définir et développer la technologie. Cockurn et Ormford (1993) dégagent également une perspective de genre dans l'analyse de la construction sociale des technologies, en mobilisant les concepts utilisés par les auteurs qui analysent la construction sociale des sciences et des technologies (Pinch et Bijker, 1990). L'ethnicité et la culture sont également envisagées dans la construction d'un rapport sexué à la technologie. Ces approches constructivistes considèrent la relation entre genre et TIC comme une construction mutuelle. Cette perspective rejoint la sociologie des usages et y introduit la dimension du genre.

Le potentiel des TIC en termes de changement sociétal dans les pays en développement est également au cœur de nombreuses recherches depuis plus d'une vingtaine d'années. Hafkin (2006), pionnière dans ce domaine, adopte une double perspective dans ses travaux, le développement et le genre, pour démontrer, à travers de nombreux exemples empiriques, comment les TIC peuvent être des outils importants susceptibles de contribuer à promouvoir 
TIC et genre : des regards multiples

l'égalité entre les hommes et les femmes et à favoriser l'autonomisation croissante des femmes. Les TIC apportent de nouvelles opportunités aux femmes, dans de nombreux domaines: l'amélioration des revenus, la conscience de leurs droits, leur bien-être et celui de leur famille, la confiance en soi, l'envie d'apprendre, etc. Les bilans de l'impact des TIC sur la vie des femmes africaines restent cependant mitigés. Dans leurs travaux, Buskens et Webb (2009) constatent que la capacité d'autonomisation et la promotion de l'égalité entre les hommes et les femmes viennent toujours buter sur des normes de genre discriminatoires. Un potentiel de changement peut être enclenché mais seulement si ces normes sont mises au second plan.

De nombreux travaux autour de l'accès aux TIC se penchent aussi sur les usages. Dans une recherche récente consacrée à la fracture numérique, Brotcorne et al. (2011) étudient les risques et les opportunités associées à différents profils types d'utilisateurs des TIC, ceci en termes d'intégration et de participation à la société de l'information. La diversité des retombées liées à l'utilisation des TIC montre qu'il n'y a pas d'emblée une relation de cause à effet entre l'usage des TIC et de meilleures opportunités ou performances dans les différentes sphères constitutives de l'intégration et de la participation sociales ${ }^{1}$. Dans cette même étude, les auteurs évaluent le degré de vulnérabilité ${ }^{2}$ des divers profils d'utilisateurs. Cette évaluation de la vulnérabilité se base sur plusieurs critères: le degré d'autonomie, les compétences, le support disponible, les comportements à risques, la valeur symbolique et le sens accordés aux TIC, la capacité de faire des choix. Dans cette approche du caractère vulnérable des situations d'utilisateurs des TIC, la variable la plus discriminante se révèle être le genre, à la défaveur des femmes qui sont plus nombreuses que les hommes dans les profils types d'utilisateurs associés à des niveaux de vulnérabilité plus importants.

Entre théorie et pratique, la perspective du cyberféminisme (Haraway, 1991 ; Butler, 1990 ; Plant, 1997 ; Adam, 1998, Kember, 2003) est également une nouvelle manière de penser la place et le pouvoir du genre dans un monde où les TIC sont devenues des réalités incontournables du quotidien. Les débats

1Dans cette étude, cinq sphères constitutives de l'inclusion sont examinées, en interaction avec l'usage des TIC : les activités sociales (les interactions avec la famille ou les amis, ou à l'intérieur d'un groupe culturel ou d'une communauté), les activités productives (l'engagement dans une activité productive, comme le travail rémunéré, l'éducation ou la formation), les activités politiques ou citoyennes (la participation citoyenne et sociale), les activités de consommation (privé/public) et le développement personnel (l'autonomie et l'estime de soi).

2 Dans l'étude, le concept de vulnérabilité désigne la probabilité de voir sa situation ou ses conditions de vie se dégrader. II intègre à la fois, une menace - liée à l'imposition d'une norme sociale propre à la société de l'information - et une capacité de réaction - liées à des capacités au sens défini par Amartya Sen (2000). 
féministes mettent en avant d'une part, le monopole des hommes sur la technologie comme constituant une source importante de pouvoir et d'autre part, le manque de compétences technologiques des femmes comme un élément important de leur dépendance à l'égard des hommes. Les approches féministes d'internet ont mis en avant une double vision, faite de craintes et d'espoir. D'un côté, une approche d'Internet plus « technique " y voit un espace masculin et d'un autre côté, une approche d'internet en termes de medium plus que de technologie y voit une opportunité de nouvelles solidarités et d'interactions entre femmes, y compris une possibilité de participation plus grande à la vie publique et citoyenne. Plus encore, les féministes inspirées par les théories postculturalistes développent le projet utopique d'un monde qui dépasserait l'opposition binaire des rapports de genre. Les cyberféministes espèrent que, dans le cadre d'Internet, les frontières entre la technologie et l'humain et entre les hommes et les femmes pourraient être dépassées. Le développement des technologies offrirait de nouvelles possibilités de se libérer du patriarcat. Ce ne sont pas seulement les technologies de l'information et de la communication mais aussi les développements de la biotechnologie qui ont une influence sur la vie, le travail et le corps des femmes Des études empiriques indiquent que ni l'ensemble des craintes, ni la totalité des espoirs ne se sont concrétisés. Dans ses travaux, Carstensen (2009) dresse une image hétérogène des relations de genre sur le web 2.0. Elle constate d'une part un renforcement des représentations stéréotypées de la masculinité et de la féminité, mais aussi l'expérimentation de diverses identités de genre, et la possibilité de politiques "queer », c'est-à-dire des mouvements qui remettent en cause les catégories d'identité sexuelle (identité de genre et orientation sexuelle), l'approche queer refusant l'enfrmement dans des «prisons identitaires $"$.

Les contributions à ce numéro de la revue tic\&société traversent cet ensemble de perspectives sur la relation entre TIC et genre. Les articles d'Isabelle Collet et de Gérard Valenduc développent des hypothèses autour de la place des femmes dans les métiers des TIC, partant des choix d'orientation et suivant, ensuite, le fil des carrières.

En effet, choisir l'informatique comme filière de formation initiale reste peu fréquent pour les jeunes filles. Depuis plusieurs décennies, et dans la plupart des pays européens, elles restent peu nombreuses dans les filières scientifiques et techniques, et encore plus dans les filières informatiques. L'article d'Isabelle Collet cherche à comprendre ce qui peut expliquer ce différentiel persistant, voire croissant, dans beaucoup de pays. Après avoir examiné, dans une approche comparative européenne, une sélection de données récentes, l'auteure développe l'hypothèse selon laquelle les choix d'études et l'exercice 
d'une profession sont des pratiques qui sont toujours médiées par un système symbolique. Ce dernier est un mélange de représentations de la réalité et d'imaginaire. Dans le cas de l'informatique, cet imaginaire a une influence très importante, mais différenciée selon le sexe (Collet et Mosconi, 2010). La dimension de genre de cet imaginaire de l'informatique est étudiée finement à travers, d'une part, l'analyse d'entretiens et, d'autre part, des références à des livres et des films de science-fiction dont on sait qu'ils sont lus par les informaticiens. L'article examine ensuite l'hypothèse de Huteau (1982) qui confronte représentation de soi et représentation de métier. Isabelle Collet montre que, dans le cas des étudiants en sciences, l'écart entre la vision de soi et le prototype de métier est plus grand dans le cas des filles que celui des garçons, même lorsque leurs usages de l'ordinateur sont identiques. Elle montre ensuite comment l'imaginaire des années 1980 s'est actualisé dans les représentations d'aujourd'hui dominées par l'image d'un homme pris dans une relation exclusive avec l'ordinateur. La perspective de l'auteure permet de dépasser l'idée, historiquement ancrée, qu'il existerait une incompatibilité fondamentale, quasi naturelle, entre les femmes et les sciences ou entre les femmes et les techniques (Mathieu, 1991; Ortner, 1998 ; Tabet, 1998), en démontrant une double construction sociale, celle de l'image des femmes et celle de l'image des sciences. Ces constructions sociales produisent des stéréotypes de genre en ce qui concerne le rapport à la technique et à certains champs professionnels.

L'article de Gérard Valenduc "suit " le parcours de femmes qui ont fait le choix de la filière informatique, qu'il s'agisse de la formation initiale ou de la formation continue. Le constat de départ est le même que celui de l'article d'Isabelle Collet: la minorité constante et généralisée des femmes dans les filières d'enseignement informatique et dans les métiers des TIC. L'auteur analyse ensuite la nature et l'évolution des portefeuilles de compétences associés aux divers métiers des TIC ainsi que les trajectoires professionnelles propres à ces métiers. II met en évidence, à chaque étape des parcours professionnels -acquisition des compétences, déroulement des carrières, entrée/sortie de la branche d'activités - le caractère sexué de la gestion des métiers des TIC, dont la culture professionnelle mâle n'est que la face émergée de l'iceberg des inégalités de genre au sein de cet univers professionnel.

L'article de Clementina Casula s'intéresse à l'enracinement ou l'encastrement social des inégalités numériques. Partant du constat de la persistance, voire l'accentuation, des disparités dans l'appropriation des TIC entre les hommes et les femmes en Italie, l'auteure tente de comprendre quels peuvent être les facteurs qui expliquent cette situation. Elle montre comment le rapport spécifique que les Italiennes entretiennent avec les TIC est imbriqué 
dans un contexte social et institutionnel fondé sur ce que l'auteure appelle un système "sexe/genre » fondamentalement inégalitaire. Le type de rapport aux TIC détermine une place dans la société de l'information qui est tantôt caractérisée par l'auto-exclusion, tantôt par l'exclusion partielle, tantôt par l'intégration complète, ce dernier scénario étant minoritaire. A partir d'entretiens réalisés avec une quarantaine de femmes engagées dans des formations aux TIC, Clementina Casula met en évidence trois types de rapport à ces technologies, chacun étant façonné par une identité et un rôle de genre spécifiques. Dans une configuration traditionaliste qui confère des identités et des rôles de genre non remis en question, les TIC se présentent comme des objets étrangers de l'univers féminin et les femmes apparaissent comme des "immigrantes numériques » (Gasser et Palfrey, 2008) débarquant, avec appréhension et méfiance, dans un dominion mâle (Turkle, 1997). Dans une configuration plus émancipatrice, les femmes italiennes se détachent des hiérarchies patriarcales et des identités et rôles de genre assignés. Elles développent alors un rapport confiant et gratifiant aux TIC qui se révèlent être des garants de liberté et un levier à l'élaboration d'un système " sexe/genre " plus égalitaire et équilibré dans la société italienne. Enfin, la troisième configuration se situe entre les deux précédemment évoquées. Elle concerne la majorité des femmes adultes italiennes pour qui le rapport aux TIC est surtout marqué par la frustration et le stress. À l'étroit dans leur rôle et leur identité de genre, ces femmes vivent une relation ambivalente et inconfortable avec les TIC qui rend compte à la fois de l'enracinement social des pratiques numériques mais aussi de la force de changement que représentent les TIC (Wajman, 1991). Pour conclure, l'auteure développe ce lien bidirectionnel dans le rapport entre la technologie et la société.

L'article de Baba Wame est également une réflexion sur l'enracinement social des usages des TIC. II propose un regard anthropologique fouillé sur les pratiques des femmes camerounaises dans un champ particulier d'usages, celui de la recherche d'un conjoint. L'auteur analyse de manière détaillée les dimensions du cadre d'usages (Flichy, 2008) de ces femmes camerounaises, d'origine sociodémographique très diversifiée. II décrit d'abord le territoire d'usages (Beauvisage, 2007) c'est-à-dire comment, pratiquement et symboliquement, cette recherche de l'âme sœur s'encastre dans un environnement quotidien : l'entourage de la "tchatteuse », le fonctionnement des points d'accès, l'encadrement des apprenantes, les dispositifs pratiques (webcam, fiches personnelles...), l'activité commerciale qui se développe autour des usages de ces femmes. Il observe également l'évolution des pratiques des femmes camerounaises en recherche du partenaire idéal via Internet : l'itinéraire qui les mène aux sites de rencontres, leurs préférences, les habitudes qui se mettent en place. L'auteur analyse longuement la place et le sens de cet usage 
TIC et genre : des regards multiples

particulier, à la fois dans sa dimension individuelle (changer sa vie, améliorer ses conditions d'existence) et dans sa dimension sociale (changer la vie de sa famille, s'inscrire dans un autre type de rapport homme/femme et un autre rapport ethnique). II démontre également les risques individuels, notamment l'exploitation économique et sexuelle.

Le type d'usage des femmes camerounaises, décrit dans l'article de Baba Wame, ne suggère ni une plus grande égalité entre les hommes et les femmes, ni une autonomisation croissante des femmes. Néanmoins, à travers l'analyse de cet usage spécifique des TIC, comme dans l'article de Clementina Casula, ces technologies apparaissent constituer des leviers potentiels d'une redéfinition des rapports de genre.

Aucune de ces perspectives théoriques ne peut toutefois, à elle seule, expliquer les écarts qui existent entre les hommes et les femmes dans le rapport aux TIC. De plus, le caractère universel de certaines hypothèses contraste avec des situations différenciées d'un pays à l'autre. De nombreuses questions demeurent donc ouvertes.

\section{Références bibliographiques}

ADAM A., 1998, Artificial knowing: Gender and the Thinking Machine, New York, Routledge.

BEAUVISAGE T., 2007, « Mesurer l'activité sur ordinateur. Enjeux et méthodes », Réseaux, vol. 25, n¹45-146, Paris, pp. 45-80.

BROTCORNE P., DAMHUIS L., LAURENT V., VALENDUC G., et VENDRAMIN P., 2011, Diversité et vulnérabilité dans les usages des TIC - La fracture numérique au second degré, Gent, Academia Press.

BUSKENS I. et WEBB A. (eds), 2009, African Women and Icts, London, Zed Books.

BUTLER J., 1990, Gender Trouble, Feminism and the Subversion of Identity. Thinking Gender, New York, Routledge.

CARSTENSEN T., 2009, "Gender Trouble in Web 2.0 : Gender Relations in Social Network Sites, Wikis and Weblogs, ", International Journal of Gender, Science and Technology, Vol. 1, $\mathrm{n}^{\circ} 1$, pp. 106-127

http://genderandset.open.ac.uk (consulté le 14.2.2011)

COCKBURN S. et S. ORMFORD, 1993, Gender and Technology in the Making, London, Sage. 
COLLET I. et N. MOSCONI, 2010, «Les informaticiennes : De la dominance de classe aux discriminations de sexe ? » Nouvelles questions féministes, vol.19, $n^{\circ} 2$, pp. $100-113$

FLICHY P., 2008, « Technique, usage et représentations », Réseaux, vol. 26, n 148-149, Hermès Lavoisier, Paris, pp. 147-174.

GASSER U. et J. PALFREY, 2008, Born Digital - Connecting with a Global Generation of Digital Natives, CITY, Perseus Publishing.

HAFKIN N. J. et S. HUYER (eds), 2006, Cinderella or Cyberella, Empowering Women in the Knowledge Society, Bloomfield, Kumarian Press.

HARAWAY D., 1991, Simians, Cyborgs, and Women, New York, Routledge.

HUTEAU M., 1982, « Les mécanismes psychologiques de l'évolution des attitudes et des préférences vis-à-vis des activités professionnelles », $L^{\prime}$ 'orientation scolaire et professionnelle, vol.11, n², pp. 107-125.

KEMBER S., 2003, Cyberfeminism And Artificial Life. New York, Routledge.

MATHIEU N.-C., 1991, L'anatomie politique : Catégorisation et idéologie du sexe, Paris, Edition Côté-femmes.

ORTNER S., 1998, " Is female to male as nature is to culture », dans Joan Landes (dir), Feminism: The public and the private, Oxford, Oxford University press, pp. 21-45.

PETERSON H., 2010, « The Gendered Construction of Technical SelfConfidence : Women's Negotiated Positions in Male-Dominated, Technical Work Settings », International Journal of Gender, Science and Technology, Vol. 2, n 1. http://genderandset.open.ac.uk (consulté le 14.2.2011).

PINCH T. J. et W. E. BIJKER, « The Social Construction of Facts and Artefacts. Or How the Sociology of Science and the Sociology of Technology Might Benefit Each Other » in Bijker W.E., Hughes, T.P. et T.J. Pinch (eds), 1990, The social construction of technological systems: new directions in the sociology and history of technology, Cambridge MA, MIT Press, pp. 17-50.

PLANT S., 1997, Zeroes + Ones : Digital Women and the New Technoculture, New York : Doubleday.

ROYAL C., 2009, Gendered spaces and digital discourse : Framing women's relationship with teh internet, VDM Verlag.

SEN A., 2000, Repenser l'inégalité, Seuil, Paris. 
TIC et genre : des regards multiples

TABET P., 1998, La construction sociale de l'inégalité des sexes : Des outils et des corps, Paris, l'Harmattan.

TURKLE S., 1997, Life on the Screen, Identity in the Age of the Internet, NewYork, Toutchstone.

VENDRAMIN P., 2004, Parcours professionnels dans les métiers des TIC, Namur, Rapport pour le Fonds social européen et la Région wallonne.

VENDRAMIN P., 2005, « TIC : écarts entre les hommes et les femmes en Europe ", Actes du colloque Les femmes face aux sciences et technologies de l'information et de la communication, Paris, 5/12/2005.

VENDRAMIN P., VALENDUC G., 2006, « Fractures numériques, inégalités sociales et processus d'appropriation des innovations », Terminal, $n^{\circ}$ 95-96, Paris, L'Harmattan, pp. 137-154.

WAJCMAN J. 1991, Feminism Confronts Technology, Polity Press. 